\title{
Environment Understanding: Robust Feature Extraction from Range Sensor Data
}

\author{
Antonio Romeo and Luis Montano \\ Departmento de Informática e Ingeniería de Sistemas. Universidad de Zaragoza \\ Instituto de Investigación en Ingeniería de Aragón, I3A \\ c/ María de Luna 1, E 50018 Zaragoza, Spain \\ Email: \{romeo,montano\}@unizar.es
}

\begin{abstract}
This paper proposes an approach allowing indoor environment supervised learning to recognize relevant features for environment understanding. Stochastic preprocessing methods in combination with either of usual pattern recognition schemes are used. Preprocessing method treated is a combination of the Principal Components Analysis and the Fisher Linear Discriminant Analysis well adapted to the sensorial information and to the kind of environments considered. The supervised method is applied to the raw range data obtained from typical indoor environments, obtaining good recognition performances without geometrical feature extraction, allowing its real time implementation.

Our work focuses on the preprocessing method, giving a geometrical interpretation of their main components, and analyzing their robustness to shape distortions and scale changes.
\end{abstract}

\section{INTRODUCTION}

Most of objectives in mobile robotics (i.e. self-localization, map building, navigation and place recognition) uses geometrical and spatial reasoning. To achieve this, a kind of environment description or map is required. Two basic types of maps are used for global navigation: on the one hand, precise metric maps are suited when precise robot localization is required. Moreover, SLAM deals concurrently with map generation and precise robot localization using a probabilistic framework [1]. On the other hand, topological approaches use an adjacency graph that represents significative locations and its connections [2][3][4]. These maps are more compact and less complex than metric maps and require a place recognition module for global robot localization. Often, hybrid solutions that combines metric and topological map are performed in order to obtain advantages of both approaches [5].

Independently of the map kind, polygonal environments are usually assumed and geometrical features as straight lines and/or their geometrical compounds (e.g. corners, doors, ceilings) are required in order to construct maps without artificial landmarks addition [6]. Hard computational effort is needed to obtain these geometrical features from sensor raw data and maintain a consistent map.

But environment descriptions in geometrical terms are not the unique possibility for our purposes. Appearance-based methods propose alternative environment descriptions that use some descriptive/symbolic features obtained directly from the sensor raw data, arising high level geometrical feature extraction and so, reducing drastically the gap between sensor raw data and spatial reasoning. These methods have been applied mainly to image analysis for face recognition purposes [7], but also they are used by robotics community in place recognition [8], robot localization [9] [10] and navigation [12]. Applications in this area cope with the problem associated to the high dimensionality of input image data. All previous approaches perform the image analysis in a stochastic framework. In particular, they use Principal Component Analysis (PCA) as main method for project the sensorial space onto less-dimensional one. However, there are few works that use appearance methods applied to rangefinder raw data. Crowley in [13] uses appearance methods only for localization purposes in a local metric map. Recently, Burgard et al. [11] use an alternative non-statistical (heuristic) appearance method combined with AdaBoost algorithm for place (rooms, doorways and corridors) recognition purposes without classic walls geometric features extraction. In any case, once raw data are processed, some recognition technique must be applied for feature recognition purposes. In [15] we can find a classical bayesian programming technique for place recognition for non-distorted environments.

In our work we claim the use of appearance-based methods applied to the raw data (pairs $(d, \alpha)$ ) obtained from a laser rangefinder sensor. Robot will navigate in a typical maze-like context (see figure 1), in which navigation across corridors represents the most important percentage of navigation time. As the system is able to learn and recognize features such as: Diaphanous corridor ( $\|$ ), End of corridor ( $\square$ ), T-intersection ( $\top$ ), Turn to left ( $\neg$ ), Turn to right ( $\ulcorner$ ), Left hand $(\dashv)$, and Right hand $(\vdash)$, we can obtain a topological representation of the environment allowing a further reasoning on it to localization or navigation. In addition, a robust environment recognition system able to recognize that compound features, no matter their size (i.e. width and length of corridors) or whether they are irregular (i.e. non-straight walls in a cave, non-polygonal scenarios) is very useful due to its generality. A such system is the target of this paper.

In order to recognize these features from raw sensor data, some kind of preprocessing is needed. We propose here to 
apply a statistical analysis of input data (a kind of training procedure), focusing our attention on two methods that project the input space onto a lower dimensional one. The process works in two steps: the first one consists of a Principal Component Analysis (PCA): an unsupervised appearance-based technique; the second one, applies a Linear Discriminant Analysis (LDA): a supervised method that enhances the discrimination capacity in order to recognize the features. Based on this information, it's possible to build a topological map, provide place recognition capability and make decisions for navigating across the environment. Also, in order to provide environment understanding, we'll give a geometric interpretation of the obtained components in shape terms.

The remainder of this paper is organized as follows: in section II we present PCA and LDA preprocessing methods, giving interpretation for the resulting main components in shape terms. In section III a bayesian scheme for shape recognition is described. In section IV, we analyze the robustness of preprocessing methods proposed in II, increasing it for distorted and scaled environments and providing the complete recognition algorithm. Finally, in section $\mathrm{V}$ we'll present some experimental results.

\section{Processing Rangefinder RaW Data: Principal COMPONENT AND LINEAR DISCRIMINANT ANALYSIS}

The method developed in this paper allows to obtain the features mentioned in the previous section, directly from the raw rangefinder information. The goal is to transform this information into another space in which the compound features can be easily recognized in a robust way, in spite of their size (scale) or their distortion. For this purpose a Principal Component Analysis and then a Linear Discriminant Analysis is achieved.

\section{A. Principal Component Analysis}

The aim of Principal Component Analysis [14] is to transform the input space in a new space whose elements are

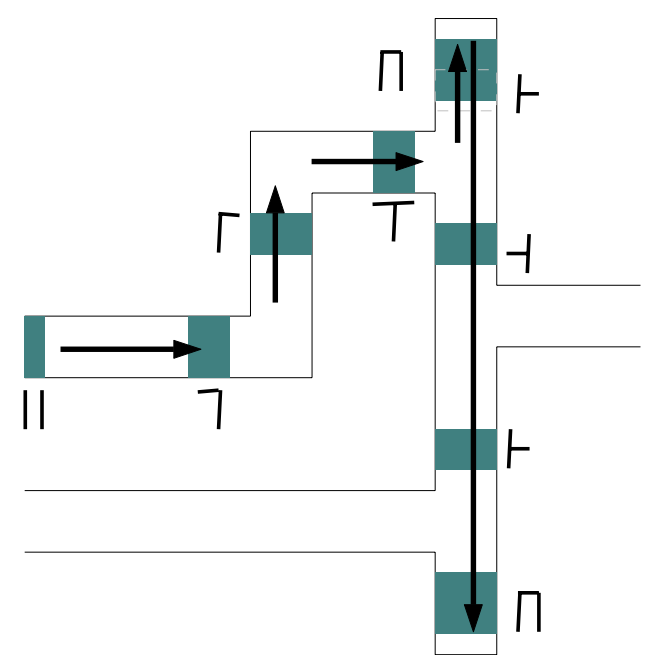

Fig. 1. Example of ideal simulated scenario and features. uncorrelated (covariance matrix will be diagonal). In other words, PCA performs a transformation in order to obtain a new set of orthogonal axis in such a way that variance is maximum. This process treats two important operations in all perception processes: feature extraction and feature selection. PCA performs feature extraction in the sense of enhancing certain features without a clear geometric interpretation. In order to reduce the dimensionality of the data, PCA improves a variance-based arrangement of the components in such a way that it's possible to select only majors of them.

Formally, let's $\mathbf{x}$ the data in a $\mathrm{n}$-dimensional space $\mathcal{P}$. We want to find transformed data $\mathbf{y}$ in a new space $\mathcal{P}^{\prime}$ obtained through a linear transformation $\mathcal{W}$ in which the variance is maximum:

$$
\mathbf{y}=\mathcal{W}^{t} \mathbf{x}
$$

The solution of this optimization problem shows that eigenvalues $\left(\lambda_{i}\right)$ of covariance matrix $\psi_{x}$ are the diagonal elements of covariance matrix in new space $\left(\psi_{y}\right)$, and eigenvectors of $\psi_{x}$ are columns $\phi_{i}$ of the transformation matrix $\mathcal{W}$ :

$$
\begin{gathered}
\psi_{y}=\mathcal{W}^{t} \psi_{x} \mathcal{W}=\operatorname{diag}\left(\begin{array}{llll}
\lambda_{1} & \lambda_{2} & \ldots & \lambda_{n}
\end{array}\right) \\
\mathcal{W}=\left(\begin{array}{llll}
\phi_{1} & \phi_{2} & \ldots & \phi_{n}
\end{array}\right)
\end{gathered}
$$

Elements of new space $\mathcal{P}^{\prime}$ are uncorrelated (covariance matrix is diagonal), and also, elements of $\psi_{y}$ are arranged if $\psi_{x}$ is positive semi-definite matrix. In order to apply this selection process to our identification system, we must define a threshold which represents the minimum percentage of contribution to global variance for considering a component. The reduction rate will depend of statistical characteristics of input set, and they'll be affected by noise, robot localization, environment shape and others. In this sense, PCA will present high noise sensibility because noise increments the variance of the input set, and surely will belongs to the selected group of components. So, final transform matrix $\mathcal{W}_{P C A}$ will be as follows:

$$
\begin{aligned}
& \mathcal{W}_{P C A}=\left(\begin{array}{llll}
\phi_{1} & \phi_{2} & \ldots & \phi_{m}
\end{array}\right), m \leq n \\
& \mathbf{y}_{\mathbf{P C A}}=\left(\mathcal{W}_{P C A}\right)^{t} \mathbf{x}^{\text {norm }}
\end{aligned}
$$

where $\mathbf{x}^{\text {norm }}$ is a normalized input vector (zero mean and unitary standard deviation for each component): $x_{i}^{\text {norm }}=$ $\left(x_{i}-\overline{x_{i}}\right) / \sigma_{i}$. In our case, vector $\mathbf{x}=\left(x_{1} \ldots x_{n}\right)^{t}$ contains the raw range data (181 values) belonging to one scan. Vector $\mathbf{y}_{P C A}$ will be the principal components of $\mathbf{x}$. Figure 2 shows an example that provides understanding to PCA: it shows the original raw data and its reconstruction from Principal Components that consider the $90 \%$ of the variance (10 components). It seems clear that the essential geometric information is contained in a few number of components. This fact will be utilized for reducing the information provided to the following step of the feature learning process. Note also the treatment of distance discontinuities: PCA performs clearly an interpolation that can be interpreted as a kind of low-pass filtering. 


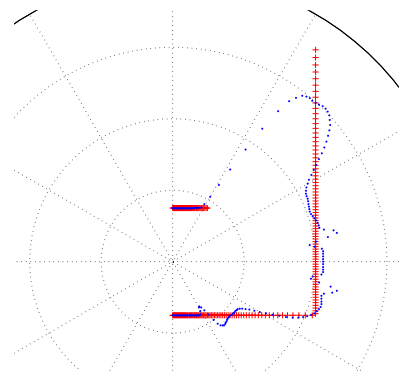

Fig. 2. Original feature (+red) and its reconstruction (.blue) using only 10 Principal Components

Let's analyze in detail the PCA, finding some geometrical interpretation in shape and/or robot localization terms. For all environments, a component will be suitable for discrimination purposes if its mean value are quite different from all others and their variance are small. Figure 3 shows mean and standard deviation values of the seven major Principal Components for each environment. These values have been obtained for 2292 different localizations in front of each feature. Localizations have been selected in such a way all the essential parts of features are visible (within a band of $0.6 \mathrm{~m}$. with respect to the central axis, an angular range of $0.1 \mathrm{rad}$ and a distance range from 3 to 1 meter with respect to nearest part of the feature). As we can see, the first component is not suitable for shape discrimination because its variance is high for all features (their ranges are clearly overlapped). However, the second component is highly suitable in order to distinguish between T-intersection ( $\top$ ) and, for example, diaphanous corridor $(\|)$.

Also, we can to analyze correlations between Principal Components and robot localization $\mathbf{q}=(x, y, \theta)$. In this sense, the first principal component is highly correlated (0.95) with $y$, and the second one is also strongly correlated (0.97) with $x$ for all closed environments $(\top, \sqcap\urcorner,\ulcorner)$, , being a detector of distance to the end of feature. We could reduce the variability of components limiting the robot location range from which the feature is recognized, i.e. with the robot centered in front of features.So, the Principal Component information can be used to improve the visibility of the feature (active perception), but this is out of the scope of this work.

The main problem of PCA components is that it is an unsupervised method and so, whereas it exhibits certain optimal properties (uncorrelated features, maximum variance, minimum reconstruction error), they may not be necessarily optimal for shape recognition (and also, for robot localization). N. Vlassis et al. in [9] propose an alternative supervised linear projection in order to optimize the performance of the robot localization task.

In summary, some positional information $(x, y)$ can be extracted from PCA components, but it isn't yet possible to discriminate the features. Figure 4 shows an alternative spatial representation of principal components 2, 3 and 4 for all the features. Thus, we can use some discriminant function for this

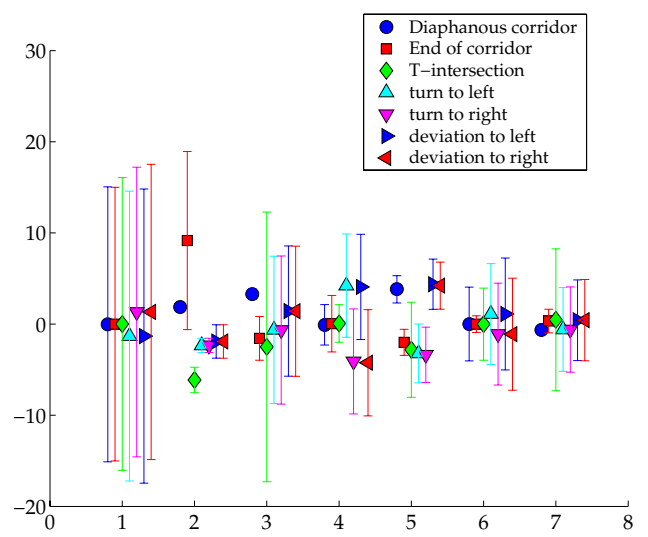

Fig. 3. Mean and standard deviation values $(2 \sigma)$ of the seven Principal Components particularized for each feature.

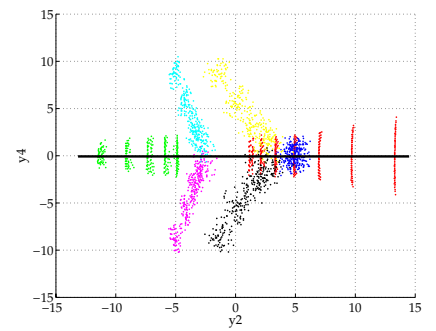

(a) View 1: $y_{2}$ and $y_{4}$

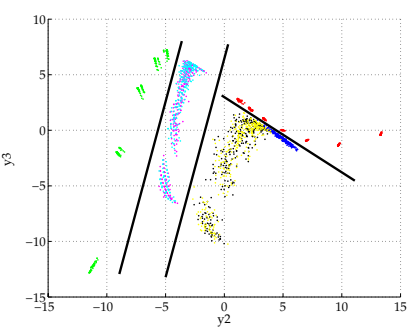

(b) View 2: $y_{2}$ and $y_{3}$
Fig. 4. Principal components 2, 3 and 4. Black axis represent the three optimal directions for feature discrimination

objective. In this sense, seeing Fig. 4, it seems clear that we can separate features using linear projections (planes). This fact allows the use of some linear discriminant functions, as proposed in next subsection.

\section{B. Linear Discriminant Analysis combined with PCA}

Fisher discriminant function [14] allows to find the linear combination $\mathbf{y}$ of input parameters that maximizes the Rayleigh ratio:

$$
\frac{\mathbf{y}^{\prime} B \mathbf{y}}{\mathbf{y}^{\prime} W \mathbf{y}}
$$

Numerator represents the variability between groups ( $W$ is the sum of covariance matrix in each group), whereas denominator corresponds to variability inside groups. The resulting linear combination emphasizes those components with high discrimination capacity. The solution is provided by eigenvectors of $W^{-1} B$. Only $q-1$ eigenvectors $\phi_{i}$ associated to the $q-1$ higher eigenvalues will be linearly independent, being $q$ the number of features to discriminate. So, the transformation matrix will be:

$$
\mathcal{W}_{L D A}=\left(\begin{array}{llll}
\phi_{1} & \phi_{2} & \ldots & \phi_{q-1}
\end{array}\right)
$$

and so:

$$
\mathbf{y}_{\mathbf{L D A}}=\left(\mathcal{W}_{L D A}\right)^{t} \mathbf{x}
$$

where $\mathbf{y}_{\mathbf{L D A}}=\left(y_{1}, \ldots, y_{q-1}\right)^{t}$, and $\mathbf{x}=\left(x_{1}, \ldots, x_{n}\right)^{t}$. Notice that columns of $\mathcal{W}_{L D A}$ represent directions in which 
discrimination capacity is optimal (in general they are not orthogonal). In Fig. 4 we can see the three directions in which we can discriminate optimally all the features.

In summary, we propose combining PCA and LDA methods in two phases process: first, we can obtain those principal components that contribute in some way to the variance (removing components that correspond to very low contributions to variance). So, all relevant information will be preserved in the new space. Covariance matrices in this space are diagonal and thus, non singular. Then, in a second phase, we can perform LDA in order to obtain discriminant components. Combining (5) and (8):

$$
\mathbf{z}_{L P}=\left(\mathcal{W}_{L D A}\right)^{t}\left(\mathcal{W}_{P C A}\right)^{t} \mathbf{x}^{\text {norm }}=\left(\mathcal{W}_{L P}\right)^{t} \mathbf{x}^{\text {norm }}
$$

where $\mathbf{z}_{L P}=\left(z_{1}, \ldots, z_{q-1}\right)^{t}$ is the discriminant components vector, and $\mathbf{x}^{n o r m}=\left(x_{1}^{n o r m}, \ldots, x_{n}^{n o r m}\right)^{t}$ is the normalized input vector. Applying this technique to a typical indoor environment (such as the Fig. 1), we obt5ain the following results: in a first phase, PCA preserve $99.9 \%$ of variance in 60 components. In the LDA phase, six discriminant components are obtained from the 60 previous selected Principal Components. Note also that first PCA phase improves a significant dimensionality reduction of the input space (from 181 to 60 ), and thus, posterior LDA process will be more efficient, as we'll see in section V. Fig. 5 shows the mean and $2 \sigma$ values of each component and feature. As we can see, these components are more suitable for shape classification than Principal Components, and it's possible intuitively to find a geometrical interpretation of the major components:

- First component can be considered as a free space detector, because it emphasizes differences between closed $(\sqcap, \top\urcorner,\ulcorner)$, and open $(\|, \dashv, \vdash)$ features.

- Second component can be interpreted as a lateral detector: it distinguishes between symmetrical, left oriented and right oriented features.

- Third component allows to distinguish between the end of corridor $(\sqcap)$ and the T-intersection $(\top)$ features.

From these three components it's possible to classify input scan raw sensor data transformed by LDA + PCA transformation matrices and, as we'll see later, the proposed method exhibits robustness when the robot is not centered in the features.

Its important to note that the proposed combination is a supervised method (as the method proposed in [9]), since LDA components are obtained under an optimization criteria in order to emphasize their discrimination capacity.

\section{Feature ReCOGNition technique}

Once obtained the Fisher's discriminant components, we can apply some pattern classification strategies in order to recognize features. Bayesian decision theory is a fundamental statistical approach to the problem of pattern classification. The method can be formally described as follows:

Let $\left\{\omega_{1}, \ldots, \omega_{q}\right\}$ the set of $q$ environments to be recognized and let $\mathbf{z}$ the feature vector (Fisher's discriminant components). The posterior probability $P\left(\omega_{j} \mid \mathbf{z}\right)$ can be obtained by

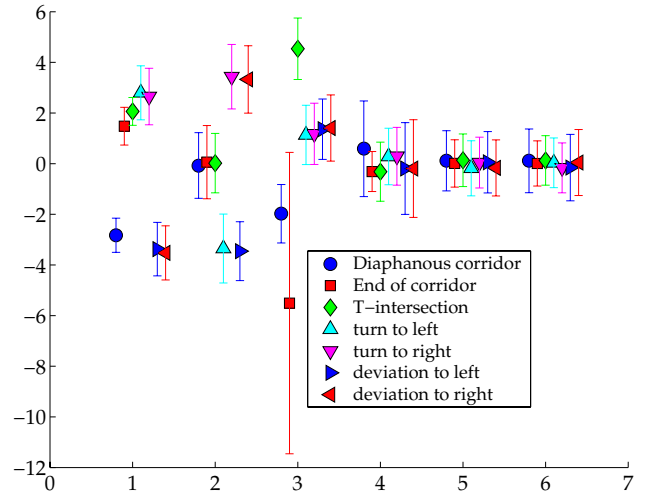

Fig. 5. Mean and standard deviation $(2 \sigma)$ values of the discriminant components.

the Bayes formula:

$$
P\left(\omega_{j} \mid \mathbf{z}\right)=\frac{p\left(\mathbf{z} \mid \omega_{j}\right) P\left(\omega_{j}\right)}{p(\mathbf{z})}=\frac{p\left(\mathbf{z} \mid \omega_{j}\right) P\left(\omega_{j}\right)}{\sum_{j=1}^{c}\left(p\left(\mathbf{z} \mid \omega_{j}\right) P\left(\omega_{j}\right)\right)}
$$

where $p\left(\mathbf{z} \mid \omega_{j}\right)$ is the likelihood, $P\left(\omega_{j}\right)$ is the prior for the $j$-th feature and $p(\mathbf{z})$ is the evidence factor. Likelihoods correspond to a normal density function. We can also integrate past time in our scheme in a simple way: the prior probability for each environment will be obtained considering the weighted average of posterior probabilities over a temporal window that consider previous $W$ steps:

$$
P\left(\omega_{j}\right)=\sum_{k=1}^{W} c_{k} P_{k}\left(\omega_{j} \mid \mathbf{z}\right)
$$

where $c_{k}$ is the weight coefficient for the $k$ previous step, $w$ is the width of temporal window (in steps), and $P_{k}\left(\omega_{j} \mid \mathbf{z}\right)$ is the posterior probability obtained at $k$ previous step. In order to provide some type of memory decay we can obtain weighting coefficients using the following exponential function:

$$
c_{k}=e^{k / W \tau}
$$

where $\tau$ represents the constant time for memory decay as a fraction of window width. This allows to filter spurious measurements, and delay the effect of entering in transition zones. Kalman Filters or Hidden Markov Models can be used as alternative ways. Finally the decision rule choose the feature with higher posterior probability.

\section{ROBUSTNESS ANALYSIS FOR THE FEATURE RECOGNITION SYSTEM}

Most of feature recognition methods are based on ideal, non-distorted geometric features. In order to extend our recognition system to real environments, it's desirable to recognize lightly distorted features. Also, shape recognition should be independent of scale factors. So, we'll analyze separately robustness to added noisy features and robustness to scale changes in following sub-sections. 


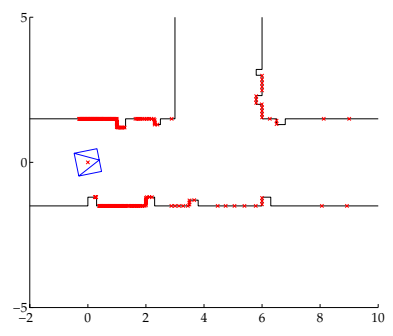

(a) Structured distortions

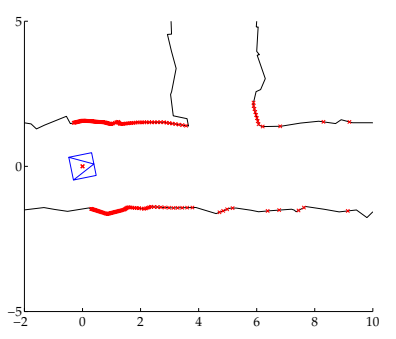

(b) Unstructured distortions
Fig. 6. Example of distortions considered.

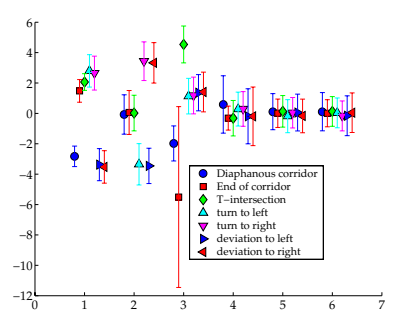

(a) Unscaled environments

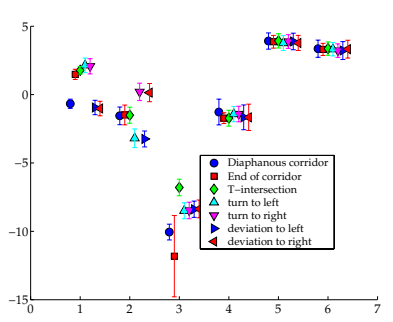

(b) $1 / 2$ scaled environments
Fig. 7. Scale factor influence in PCA + LDA transformation.

\section{A. Robustness against noise}

Sometimes, real environments exhibit little changes in their geometry with respect to initial idealizations. In some cases, these changes are referred to little features initially not considered (e.g.: frames of the doors). In other cases environments are not structured (natural scenarios) and so they don't exhibit straight lines. We can distinguish several sources of noisy features:

- Little permanent added structured features (frames of doors, windows, parts of structural columns, etc).

- Unstructured distortions of environments (i.e. corridors in caves).

- Non reflected ray and sporadic bad reflections.

- Noise over measures introduced by sensor.

Fig. 6 shows the two kind of distortions above considered. It's well known that regularization allows to reach a tradeoff between generalization capacity and overfitting. Bishop in [16] proves that training with noise is equivalent to Tikhonov regularization.

\section{B. Robustness to scale changes}

In order to analyze the size/scale dependencies, we compare the results of applying PCA+LDA transform matrices obtained from non-scaled environments to scaled non-distorted environments (see figure 7). In order to analyze the scale factor influence, we can rewrite (9) for scaled environments as:

$$
\mathbf{z}_{L P}=\left(\mathcal{W}_{L P}\right)^{t} \Sigma^{-1}(K \mathbf{x}-\overline{\mathbf{x}})
$$

where $K$ is the scale factor, $\Sigma^{-1}$ is a square diagonal matrix formed by the inverse of variance for each input component for the train set and $\overline{\mathbf{x}}$ is the mean input raw scan vector of the training set. We can write seven linear equations (one for each feature) that relate the scale factor $K$ with the mean input vector for each feature. Let's $\overline{\mathrm{x}^{j}}$ the mean input vector (training set) for the feature $j$, and $\overline{\mathbf{z}^{j}}$ the mean vector of discriminant components for the same feature. The previous general expression can be particularized for the $j$ feature as:

$$
\overline{\mathbf{z}^{j}}=K\left(\mathcal{W}_{L P}\right)^{t} \Sigma^{-1} \overline{\mathbf{x}^{j}}-\left(\mathcal{W}_{L P}\right)^{t} \Sigma^{-1} \overline{\mathbf{x}}=A^{j} K+C
$$

Where $A^{j}$ and $C$ are known ( $C$ is the same for all environments and $A^{j}$ must be computed from train set for each environment). For example, considering the diaphanous corridor case $(j=1)$, we can compute the mean input vector for the train set, and we will obtain the following values for $A^{j}$ and $C$ vectors:

$$
\begin{aligned}
A^{j} & =(-4.31,2.96,16.15,3.72,-7.61,-6.49)^{t} \\
C & =(1.49,-3.04,-18.12,-3.13,7.72,6.60)^{t}
\end{aligned}
$$

From figure 7 we can note that mean values for $6^{\text {th }}$ discriminant component (a component without a clear geometrical interpretability) are approximately the same for all the features, and consequently, we can use it for scale factor detection. Once the scale factor value $K$ is known, we can reject its influence simply affecting the input raw vector by $K^{-1}$ and then applying the recognition protocol described in previous sections.

The overall process detailed in previous sections can be described by the following recognition algorithm:

TABLE I

RECOGNITION ALgORITHM

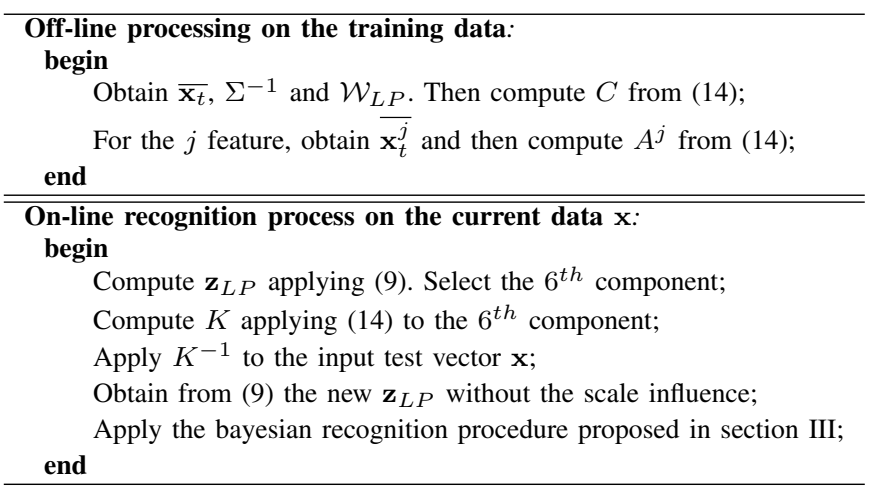

\section{EXPERIMENTAL RESULTS}

In order to evaluate the performance of our recognition system, we have developed two different experiments: the first one has been performed over a simulated ideal scenario, whereas the second experiment has been performed in a real (and distorted) one. In advance, we wish to note the low computational cost of the proposed method: $92 \mu \mathrm{sec}$ for the preprocessing PCA+LDA product (9) applied to each rangefinder scan, over a Pentium IV processor $(3.4 \mathrm{GHz})$ executing MATLAB.

\section{A. Test over simulated scenarios}

The training set for parameter extraction is formed by 2904 laser scans of the seven selected features obtained from simulated environments. For testing purposes, we have designed 
TABLE II

PERCENTAGE OF CORRECT RECOGNITION FOR EACH FEATURE IN THE SIMULATED IDEAL SCENARIO

\begin{tabular}{|c|c|c|c|c|c|c|}
\hline$\|$ & $\sqcap$ & $\top$ & $\neg$ & \ulcorner & $\dashv$ & $\vdash$ \\
\hline $99.2 \%$ & $100 \%$ & $98.4 \%$ & $100 \%$ & $100 \%$ & $98.5 \%$ & $78.7 \%$ \\
\hline
\end{tabular}

an experiment over a simulated scenario (Fig. 1) in which we can find all the features treated in this work. The experiment as been designed as follows:

- Recognition system operates each 0.5 meters along the corridor axis.

- For each axial position, we test the system in 25 combinations of transversal position (-0.1 to 0.1 meters) and orientation ( -0.04 to 0.04 radians).

Fig. 1 shows the sequence of places (topological path) visited. Dark areas represent the regions where our system must identify (these areas exhibit training compatibility) the feature in front of the robot. Note that the shape recognition procedure is very conditioned by training set selection. In our case, we have used a restrictive training policy: only when the features are sufficiently near, the robot must be able to recognize them. Anyway, if the robot is too near to the features, some essential parts of them can disappear of the sensing area. For this reason, out of dark areas, the recognition system could classify the feature as unknown. However, in such areas adjacent to the above mentioned, the system recognizes the feature, exhibiting in this form its robustness. Table II shows the recognition performance for the different features included in the proposed path. Note the low value for the right hand feature. It's due to a front-end apparition when the system is recognizing the last right hand. In other words, two features $(\sqcap$ and $\vdash)$ are visible at same time and consequently this situation is classified as unknown. These kind of problems require a different treatment to separate the sensed features, and it's being treated in an ongoing work. As we can see, the proposed method has a potential discriminant power for its use in maze-like scenarios.

\section{B. Test over real scenarios}

In order to evaluate the performance of our recognition system, we have used the data collected from the A level of the CMU - Newell-Simon Hall by a Sick PLS laser range finder mounted on GRACE robot. This data set was obtained from the Robotics Data Set Repository (Radish) [17]. Thanks go to Nick Roy for providing this data.

Fig. 8 shows the path visited. Grey narrow areas represent regions where our system should identify (these areas exhibit training compatibility in position) the feature in front of the robot. In this sense, it should be noted that:

- Some features are quite different than the employed in training phase in the ideal simulated scenario (they present distortions or added obstacles).

- Some corridors have different width than the employed in training phase

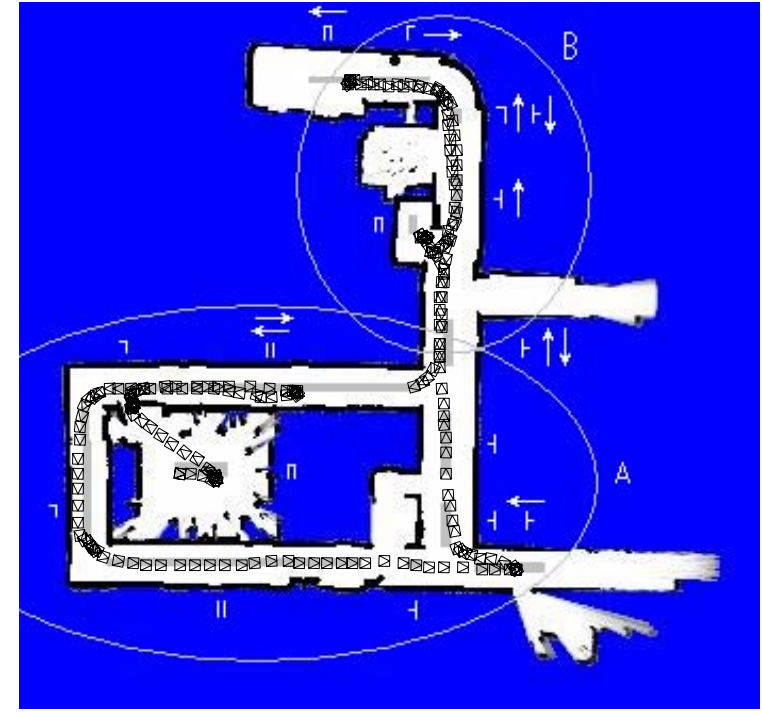

Fig. 8. Path along experiment. Grey areas indicate training compatibility zone for feature recognition.

- A relevant part of robot path is outside areas of train compatibility.

As mentioned earlier, the feature recognition procedure is very conditioned by the training set selection and so, out of mentioned compatibility areas, the recognition system could classify features as unknown. However, in such areas adjacent to the above mentioned, the system recognizes the feature, exhibiting its robustness. Table III shows the performance of recognition system for the different features included in the proposed path. The first row shows global results whereas rows 2 and 3 show results for two different areas denoted as $A$ and $B$ in Fig. 8. The reason for distinguishing two different areas in order to analyze results is that we can find an area (denoted as $B$ ) in which we observe heavy distortions in features i.e. nonpolygonal turns (see Fig. 9(a)) and also, several (compound) features are visible at same time. These compound features (see Fig. 9(b)) can be classified as unknown because its novelty. In order to improve more robustness in these cases, it should be necessary to separate the sensed features as mentioned in previous sub-section. Moreover, the robot path in this area differs a lot of the employed in training phase. On the other hand, area denoted as $A$ presents more clear (and clean) features, and we can expect results not conditioned by heavy distortions and feature compositions. Analyzing the results for this case, we can conclude that for all the features present in $A$ area $(\|, \sqcap$,$\urcorner and \dashv)$, results are very good, and reflect the suitability of our recognition procedure in order to apply it in scenarios with features different (in shape and size) to the ones used in the training phase. In addition, erroneous recognitions are concentrated in zones adjacent to ones in which some essential parts of features are lost. Figure 10 shows an example of this fact: erroneous results are concentrated in two zones, far fromo the feature and on it, as was expected (in these zones essential parts of the feature are not visible). Note also that 


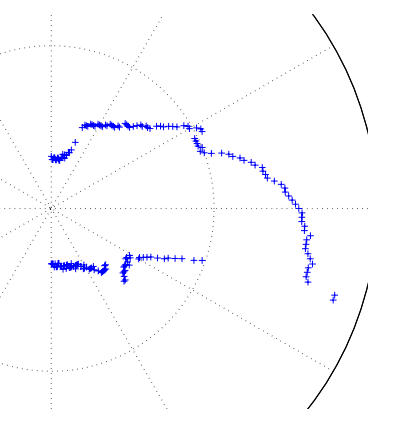

(a) distorted feature

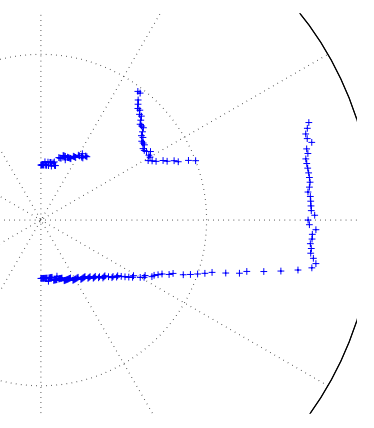

(b) compound feature
Fig. 9. Robustness of recognition procedure. Two different cases

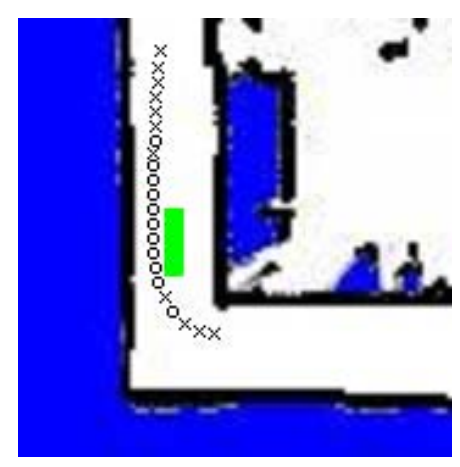

Fig. 10. Robustness example: $x$ dots represent erroneous classification whereas $o$ dots represent good classification. Green area in the center of the corridor indicates training compatibility zone for feature recognition.

the path analyzed is even out of training compatibility area, exhibiting in this form the robustness of the proposed method.

Results for the features present in $B$ area reflect above mentioned: note the low values for $\ulcorner, \vdash$ and $\dashv$ features. In the first case, the unique feature included along the path is seriously distorted, and consequently, final results reflect this fact. In the second case, distortion is provided by a walking person that passes near to the robot, and finally, for the unique $\dashv$ feature present, the robot lost visibility of essential parts of the feature.

With this experiment we wish to highlight that our method is quite robust in real environments. Notice that the training set used is based on simulated environments, but the technique is applied to another different environment and in different conditions to the applied while system learns.

\section{CONCLUSION AND FURTHER WORK}

In this work we have presented a method that provides environment recognition in terms of semantic classes, using Principal Components and Fisher Discriminant Components techniques. The environment descriptions don't need to obtain geometric features, requires low computational efforts, sinze only it's necessary to compute one matrix product $\left(\mathcal{W}_{L P}\right)$ in real time, and features are computed from the laser rangefinder raw data. The method is suitable for a topological feature recognition and for topological map building and place recognition. From the experiments, we can conclude that
TABLE III

PERCENTAGE OF CORRECT CLASSIFICATIONS FOR EACH FEATURE

\begin{tabular}{|c|c|c|c|c|c|c|}
\hline & $\|$ & $\sqcap$ & \urcorner & \ulcorner & $\dashv$ & $\vdash$ \\
\hline Global & $94.7 \%$ & $97.2 \%$ & $71.6 \%$ & $37.4 \%$ & $59.1 \%$ & $30.0 \%$ \\
\hline A area & $94.7 \%$ & $100.0 \%$ & $94.1 \%$ & - & $100.0 \%$ & - \\
\hline B area & - & $95.5 \%$ & $41.4 \%$ & $37.4 \%$ & $47.5 \%$ & $30.0 \%$ \\
\hline
\end{tabular}

the technique works in real environments and is robust to some distortions and scale changes. As a further work, we'll extend the recognition procedure to other topological features, accomplishing a specific treatment for compound features, and providing a topological localization method.

\section{REFERENCES}

[1] J.A. Castellanos, J.M. Martínez, J. Neira and J.D. Tardós, "Simultaneous Map Building and Localization for Mobile Robots: A Multisensor Fusion Approach", IEEE Int. Conf. on Robotics and Automation, ICRA'98, Leuven, Belgium, 16-21 May 1998, pp. 1244-1249.

[2] M. Mataric, "Integration of representation into goal-driven behavior-based robots". IEEE Trans. on Robotics and Automation, Vol.8 No.3, 1992, pp. 304-312

[3] B. Kuipers and Y. Byun, "A robot exploration and mapping strategy based on a semantic hierarchy of spatial representations". Robotics and Autonomous Systems Vol.8, 1991, pp. 47-63. 5.

[4] D. Kortenkamp and T. Weymouth, "Topological mapping for mobile robots using a combination of sonar and vision sensing" Proceedings of the Twelfth National Conference on Artificial Intelligence (AAAI-94), July, 1994.

[5] S. Thrun, "Learning metric-topological maps for indoor mobile Robot Localization" in Artificial Intelligence 99, (1), 1998, pp. 21-71.

[6] J.J. Leonard and H.F. Durrant-White, "Mobile Robot Localization by tracking geometric beacons" IEEE Trans. Robotics and Automation, vol. 7, no. 3, 1991, pp. 376-382.

[7] A. Martínez and J. Vitriá, "Clustering in Image Space for Place Recognition and Visual Annotations for Human-Robot Interaction" IEEE Trans. Systems, Man and Cybertnetics-Part B, vol. 31, no. 5, october 2001,pp. 669-682.

[8] I. Ulrich and I. Nourbakhsh, "Appearance-Based Place Recognition for Topological Localization," Proceedings of ICRA 2000, Vol. 2, April, 2000, pp. 1023-1029.

[9] N. Vlassis, Y. Motomura and B. Kröse, "Supervised Feature extraction for Mobile Robot Localization", IEEE Int. Conf. on Robotics and Automation, ICRA'00. San Francisco, CA, april 2000, pp. 2979-2984.

[10] B.J.A. Kröse et al., "A Probabilistic Model for Appearance-Based Robot Localization", Image and Vision Computing. no. 19, 2001, pp. 381-391.

[11] O. Martínez, C. Stachniss and W. Burgard, "Supervised Learning of Places from Range Data using AdaBoost", IEEE Int. Conf. on Robotics and Automation, ICRA'05. Barcelona, Spain, april 2005, pp. 1742-1747.

[12] S. D. Jones and J. L. Crowley, "Appearance Based Processes for Visual Navigation", IROS '97, IEEE International Conference on Intelligent Robots and Systems, Grenoble, Sept. 1997.

[13] J. L. Crowley, F. Wallner and B. Schiele, "Position Estimation Using Principal Components of Range Data", Robotics and Autonomous Systems, Vol 23, no 4, 1998, pp. 267-276.

[14] R.O. Duda, P.E. Hart, D. G. Stork, "Pattern Classification", 2nd Edition, John Wiley and Sons, 2001.

[15] A. Tapus, G. Ramel, L. Dobler, R. Siegwart, "Topology Learning and Recognition using Bayesian Programming for Mobile Robot Navigation", IEEE/RSJ IROS '04, 2004, pp. 3139-3144

[16] C. M. Bishop, 'Training with Noise is Equivalent to Tikhonov Regularization", Neural Computation, Vol. 7, Issue 1, january 1995, pp. 108-116

[17] A. Howard, N. Roy, 'The Robotics Data Set Repository (Radish)". 2003, http//radish.sourceforge.net/ 